\title{
Tratamiento endovascular en arteritis de Takayasu. Presentación de un caso clínico
}

\author{
Endovascular intervention in Takayasu Arteritis. Case report
}

\author{
Dra. Marisa Di Santo ${ }^{a}$ Dra. Érica V. Stelmaszewski ${ }^{a}$ y Dra. Alejandra Villa ${ }^{a}$
}

\begin{abstract}
RESUMEN
Una paciente de cuatro años concurrió a la Guardia por edemas e hipertensión arterial. Al momento del examen físico, se detectó disminución del pulso en el brazo izquierdo y ausente en los miembros inferiores, con diferencia de tensión arterial mayor de $10 \mathrm{mmHg}$ entre los miembros superiores. Se realizó un ecocardiograma, en el que se observó coartación grave de la aorta a nivel abdominal y disfunción ventricular. Con la sospecha de arteritis de Takayasu, se completaron estudios con angiotomografía cardíaca, que confirmó una desconexión de la arteria subclavia izquierda y estrechez grave en la aorta abdominal. El diagnóstico definitivo fue de arteritis de Takayasu. Se realizó una angioplastía con estent por Hemodinamia en la aorta abdominal, con buenos resultados posteriores. Mejoraron los pulsos de los miembros inferiores, y se obtuvieron valores normales de la tensión arterial. En el ecocardiograma, mejoró la función ventricular, y el gradiente en la zona de coartación se redujo significativamente.

Palabras clave: arteritis de Takayasu, estents, coartación aórtica, angioplastía.
\end{abstract}

\begin{abstract}
A four year old patient with no medical history was admitted to our hospital's Emergency Department, suffering from edema and hypertension. During physical examination a low pulse was detected in the left arm and a lack of pulses in lower limbs, with a blood pressure difference greater than $10 \mathrm{~mm} \mathrm{Hg}$ between both arms. An echocardiogram demonstrated severe aortic abdominal coarctation and ventricular dysfunction. Takayasu arteritis was diagnosed and cardiac angiography was performed. Disconnection of left subclavian artery and severe tightness at the abdominal aorta were confirmed. The definitive diagnosis was Takayasu arteritis. Angioplasty with stent was performed in abdominal aorta, with good subsequent results. The pulses improved in the lower limbs, and normal blood pressure values without gradient between all members were registered. The echocardiogram improved ventricular function and the gradient in the coarctation area was significantly reduced.
\end{abstract}

Keywords: Takayasuarteritis, stents, aortic coarctation, angioplasty.

http:/ /dx.doi.org/10.5546/aap.2016.e147

a. Servicio de Cardiología Infantil.

Hospital "Prof. Dr. Juan P. Garrahan".

Ciudad de Buenos Aires, Argentina.

Correspondencia:

Dra. Érica V.Stelmaszewski, eryvictoria@yahoo.com.ar

Financiamiento: Ninguno.

Conflicto de intereses: Ninguno que declarar.

Recibido: 24-8-2015

Aceptado: 18-11-2015

\section{INTRODUCCIÓN}

La arteritis de Takayasu es una vasculitis granulomatosa idiopática crónica, que afecta a la aorta y a sus ramas principales. Su descripción original data de $1908 .{ }^{1}$ Es poco común en pediatría, aunque existen numerosas publicaciones en niños y, especialmente, en adultos, que analizan la sintomatología y la presentación clínica, los métodos de estudio, el tratamiento, el seguimiento y el pronóstico. ${ }^{2-13}$

Si bien existen varias posibilidades de criterios diagnósticos, con frecuencia, se utilizan los publicados en 1990 por el Colegio Americano de Reumatología, ${ }^{13}$ quienes establecieron seis simples y prácticos criterios:

- Comienzo antes de los 40 años.

- Claudicación de una extremidad.

- Disminución del pulso arterial braquial.

- Diferencia de la presión sistólica mayor de $10 \mathrm{mmHg}$ entre los brazos.

- Soplos sobre las arterias subclavias y/o la aorta.

- Evidencias radiológicas de estenosis u oclusión de la arteria aorta y de sus ramas principales.

La presencia de tres o más de estos criterios demostró una sensibilidad diagnóstica del 90,5\% con una especificidad del $97,8 \%$.

La afectación de la aorta abdominal y de las arterias renales uni- o bilateralmente es común, lo que puede llevar a hipertensión arterial e isquemia renal. En los adultos, se describe entre un $33 \%$ y un $83 \%$ de hipertensión ${ }^{6-8}$ y la mayoría de las publicaciones pediátricas muestran la hipertensión como una de las características principales. ${ }^{4,5}$

Últimamente, los progresos en técnicas de imagen permiten diagnosticar con exactitud y contribuyen al seguimiento de este tipo de pacientes, entre los que se destacan la resonancia nuclear magnética (RNM), la angiorresonancia magnética (angio-RNM), la tomografía computarizada (TC), la angiotomografía computarizada (angio-TAC), la ecografía doppler y la tomografía de emisión de positrones (positron emission tomography; PET, por sus siglas en inglés). 
Nuestro objetivo es presentar a una paciente, cuyo diagnóstico se hizo en la etapa de remisión de la enfermedad, en la que las lesiones y las secuelas de los vasos ya estaban instaladas, y lo novedoso del caso es el tratamiento realizado (angioplastía con estent de la coartación abdominal).

Existen trabajos que comparan el tratamiento quirúrgico con el tratamiento endovascular y resaltan que los pacientes sometidos a cirugía tienen menor tasa de recurrencia, pero las complicaciones (incluso fatales) son mayores que en los pacientes que se someten a la revascularización por angioplastía. ${ }^{14,15}$

\section{CUADRO CLÍNICO}

Paciente de cuatro años, sin antecedentes patológicos, que concurrió a la Guardia del Hospital por edemas e hipertensión arterial $(128 / 87 \mathrm{mmHg})$. Se encontraba afebril. En un primer momento, se sospechó síndrome nefrítico, que se descartó con el análisis de laboratorio normal (glóbulos blancos: 10 000, 50\% de neutrófilos/40\% de leucocitos; hemoglobina: $10 \mathrm{mg} / \mathrm{dl}$; plaquetas: 329 000; albúmina: 3,9 mg/dl; creatinina: 0,5 mg/dl; urea: $34 \mathrm{mg} / \mathrm{dl}$; orina completa sin proteinuria ni hematuria).

$\mathrm{Al}$ momento del examen físico, se detectó una disminución del pulso en el brazo izquierdo y ausente en los miembros inferiores, con diferencia de tensión arterial (TA) mayor de $10 \mathrm{mmHg}$ entre los miembros superiores (TA del miembro superior derecho: $128 / 87 \mathrm{mmHg}$; miembro superior izquierdo: $110 / 79 \mathrm{mmHg}$; miembro inferior derecho: $78 / 49 \mathrm{mmHg}$; miembro inferior izquierdo: $88 / 59 \mathrm{mmHg}$ ).

FIGURA 1. Ecocardiograma: coartación grave de la aorta abdominal con gradiente de $74 \mathrm{mmHg}$

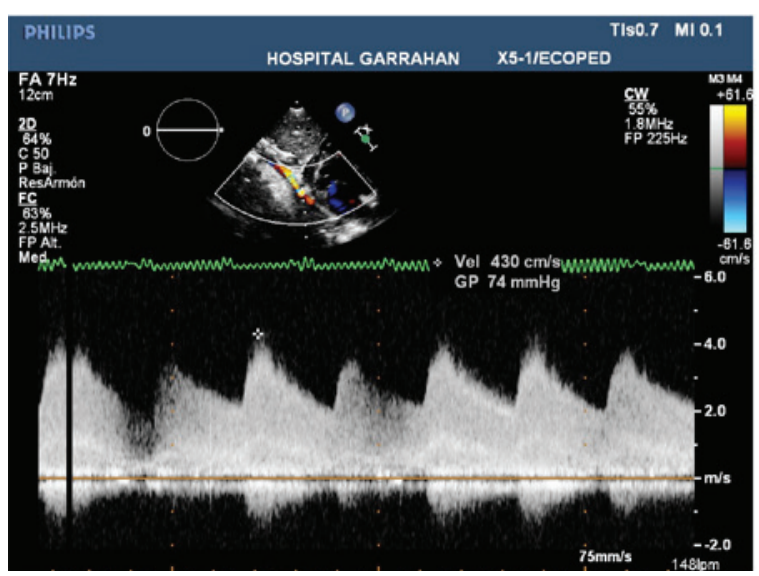

En la radiografía de tórax ( $\mathrm{Rx} \mathrm{Tx}$ ), presentaba cardiomegalia grave a expensas de cavidades izquierdas y signos de congestión pulmonar. En el electrocardiograma, se observaban trastornos difusos de la repolarización con hipertrofia del ventrículo izquierdo.

En nuestro Laboratorio de Ecocardiografía (Servicio de Cardiología), realizamos un ecocardiograma, en el que se observó coartación grave de la aorta a nivel abdominal (gradiente de $74 \mathrm{mmHg}$ con corrida diastólica) y disfunción ventricular (fracción de acortamiento de 9\%) (Figura 1). Con la sospecha de arteritis de Takayasu, se completaron los estudios con angiotomografía cardíaca (Figura 2), que confirmó una desconexión de la arteria subclavia izquierda y estrechez grave en la aorta abdominal.

El diagnóstico definitivo fue de arteritis de Takayasu en estadio de remisión (la paciente cumplía 4/ 6 criterios de 1990 del Colegio Americano de Reumatología). El Servicio de Hemodinamia Infantil (cardiólogos

FIGURA 2. Angiotomografía que demuestra una desconexión de la arteria subclavia izquierda y coartación grave de la aorta abdominal

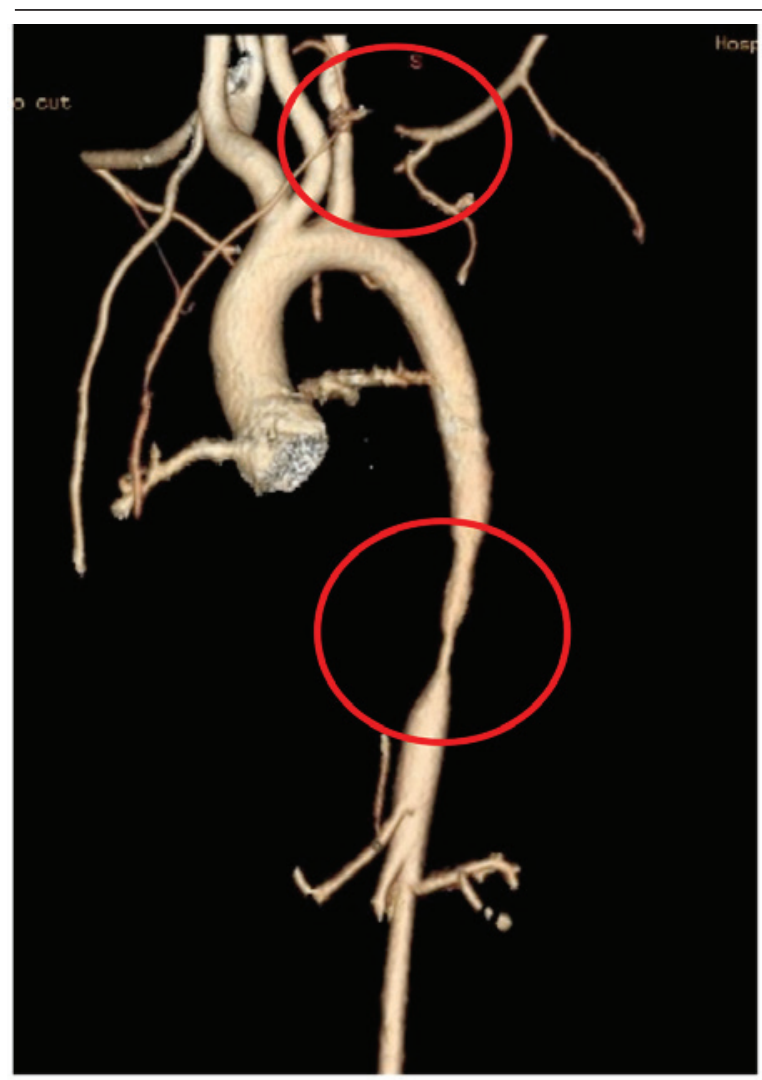


hemodinamistas) realizó una angioplastía con estent en la aorta abdominal, con buenos resultados posteriores (Figuras 3 y 4). Mejoraron los pulsos de los miembros inferiores, y se obtuvieron valores normales de TA y sin gradiente entre todos los miembros. En el ecocardiograma, mejoró la función ventricular (fracción de acortamiento de $25 \%$ ), y el gradiente en la zona de coartación se redujo significativamente (gradiente de $13 \mathrm{mmHg}$ ).

\section{DISCUSIÓN}

La arteritis de Takayasu es una enfermedad vascular sistémica progresiva y crónica, que ocasiona isquemia en los órganos vitales, incrementa la morbimortalidad, limita la supervivencia y produce un grave deterioro en la calidad de vida de los pacientes.

En una gran proporción de los pacientes, el diagnóstico se retrasa en un período de meses a años y se diagnostica, incluso, cuando la lesión arterial es extensa. La inflamación crónica puede ocasionar estenosis localizada, oclusión, dilatación y formación de aneurismas en las arterias afectadas.

Las manifestaciones clínicas inespecíficas al inicio de la enfermedad, la baja incidencia de la enfermedad y el impacto clínico de las lesiones isquémicas fortalecen la necesidad de establecer un diagnóstico temprano y oportuno.

Figura 3. Cateterismo previo a la colocación del estent, en el que se observa una coartación grave a nivel abdominal

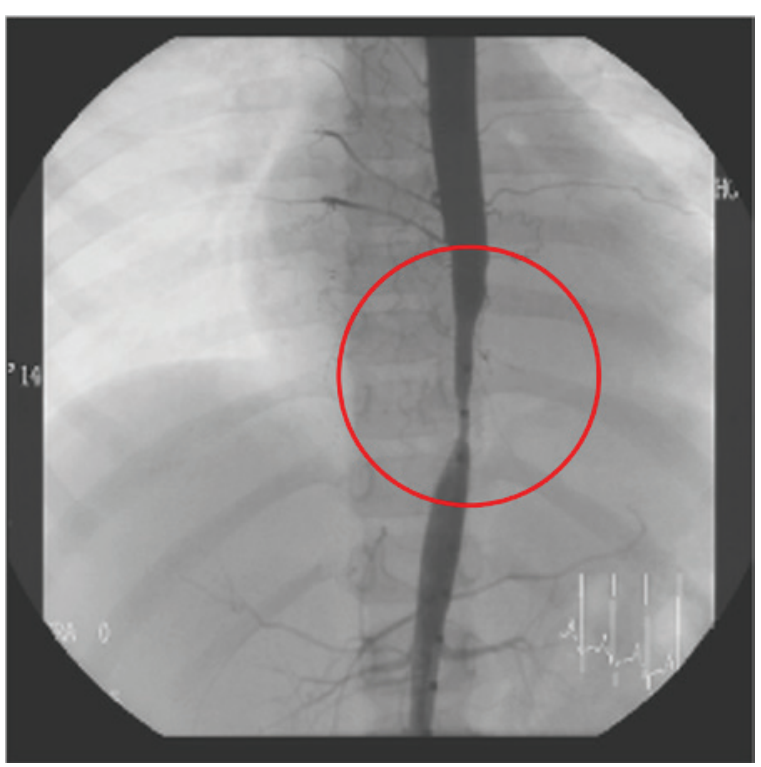

El diagnóstico precoz en la etapa aguda de la arteritis de Takayasu es difícil de sospechar. Esta etapa es el momento apropiado para el tratamiento médico antiinflamatorio (corticoidesinmunosupresores) que evite las secuelas.

El hallazgo de laboratorio más importante en la etapa aguda es el aumento de la proteína C reactiva y eritrosedimentación.

Es recomendable realizar el tratamiento de revascularización (quirúrgico vs. hemodinámico) en la etapa de remisión, en la que las lesiones ya están instaladas. En la etapa aguda, el riesgo de reestenosis y lesiones vasculares es mayor (por la friabilidad del tejido). ${ }^{14,15}$

El uso combinado de las herramientas diagnósticas tomografía, resonancia y PET ha mejorado la detección de las lesiones de forma precoz.

Los diagnósticos diferenciales incluyen otras causas de vasculitis de grandes vasos: aortitis inflamatoria (sífilis, tuberculosis, lupus eritematoso sistémico, artritis reumatoidea, espondiloartropatías, enfermedad de Kawasaki y arteritis de células gigantes), anormalidades del desarrollo (coartación de aorta, síndrome de Marfan).

El seguimiento de esta patología incluye el control con ecocardiograma doppler color cardíaco y de aorta abdominal, y, últimamente, se ha utilizado la PET. Permite la identificación temprana de todos los vasos afectados, los cuales

FIgURA 4. Cateterismo posterior a la colocación del estent, en el que se observa una mejoría de la luz del vaso

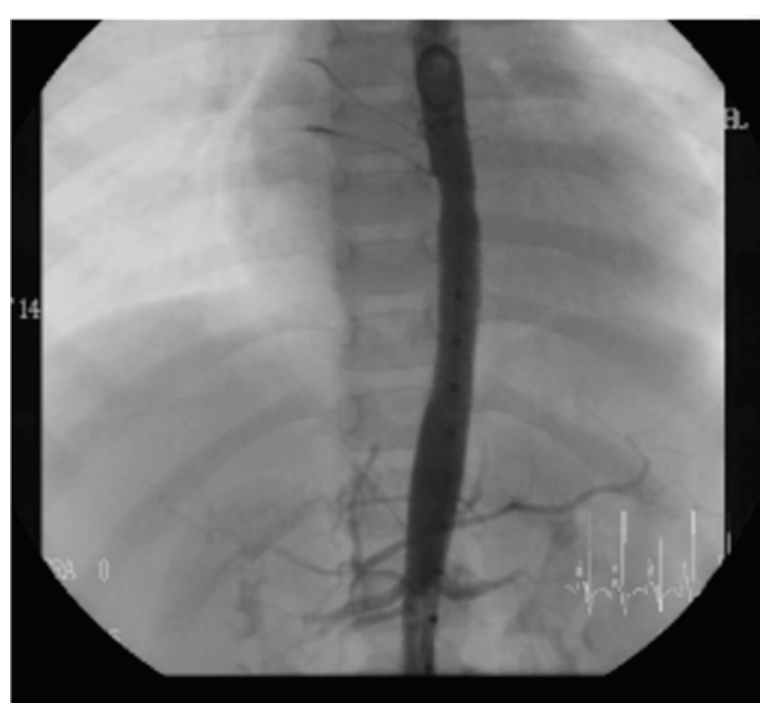


muestran, debido a la actividad inflamatoria, un aumento de la captación de F18-desoxiglucosa. Es posible, de este modo, el diagnóstico precoz de la enfermedad y el seguimiento de la respuesta terapéutica, dado que la captación disminuye con la remisión.

La arteritis de Takayasu es una enfermedad vascular sistémica progresiva y crónica, que ocasiona isquemia en los órganos vitales, limita la supervivencia y produce un grave deterioro en la calidad de vida de los pacientes. Se reporta un compromiso en las actividades de la vida diaria en el $74 \%$ de los pacientes e incapacidad laboral hasta en el $23 \%$, lo que permite comprender el impacto socioeconómico de este tipo de vasculitis. ${ }^{14}$

\section{REFERENCIAS}

1. Feld LG, Weiss RA, WeinerS, Greifer I. Takayasu's arteritis. Asymptomatic presentation in a two-year-old boy. NY State J Med 1983;83(2):229-31.

2. Saieh C, Yurlow E, Arce JD, Puga F, et al. Arteritis de Takayasu en un lactante. Bol Méd Hosp Infant Méx 1985;42(2):135-9.

3. Lacombe M. Localisations réno-aortiques de la maladie de Takayasu. Ann Chir 2002;127(4):268-75.

4. Baumgartner D, Sailer-Höck M, Baumgartner C, Trieb $\mathrm{T}$, et al. Reduced aortic elastic properties in a child with Takayasu arteritis: case report and literature review. Eur J Pediatr 2005;164(11):685-90.
5. Johnston SL, Lock RJ, Gompels MM. Takayasu arteritis: a review. J Clin Pathol 2002;55(7):481-6.

6. Mwipatayi BP, Jeffery PC, Beningfield SJ, Matley PJ, et al. Takayasu arteritis: clinical features and management: reports of 272 cases. ANZ J Surg 2005;75(3):110-7.

7. Ozen S, Ruperto N, Dillon MJ, Bagga A, et al. EULAR/ PReS endorsed consensus criteria for the classification of childhood vasculitides. Ann Rheum Dis 2006;65(7):936-41.

8. Andrew J, Al-Nahhas A, Pennell DJ, Hossain MS, et al. Non-invasive imaging in the diagnosis and management of Takayasu's arteritis. Ann Rheum Dis 2004;63(8):995-1000.

9. Valsakumar AK, Valappil UC, Jorapur V, Garg N, et al. Role of immunosuppressive therapy on clinical, immunological, and angiographic outcome in active Takayasu's arteritis. I Rheumatol 2003;30(8):1793-8.

10. Miyata T, Sato O, Koyama H, Shigematsu H, et al. Longterm survival alter surgical treatment of patients with Takayasu's arteritis. Circulation 2003;108(12):1474-80.

11. Castellanos AZ, Campos LA, Liphaus LB, Marino JC, et al. Arteritis de Takayasu. An Pediatr (Barc) 2003;58(3):211-6.

12. Fraga A, Medina F. Takayasu's arteritis. Curr Rheumatol Rep 2002;4(1):30-8.

13. Arend WP, Michel BA, Bloch DA, Hunder GG, et al. The American College of Rheumatology 1990 criteria for the classification of Takayasu arteritis. Arthritis Rheum 1990;33(8):1129-34.

14. Saadoun D, Lambert M, Mirault T, Resche-Rigon M, et al. Retrospective analysis of surgery versus endovascular intervention in Takayasu arteritis: a multicenter experience. Circulation 2012;125(6):813-9.

15. LeeGY,JeonP,DoYS, Sung K, etal. Comparison of outcomes between endovascular treatment and bypass surgery in Takayasu arteritis. Scand J Rheumatol 2014;43(2):153-61. 